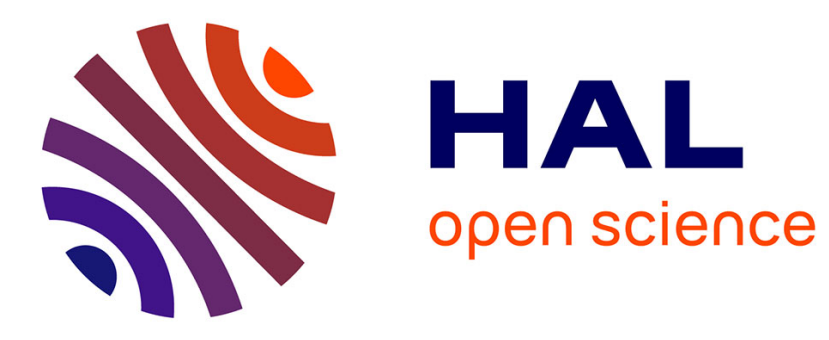

\title{
Drag Enhancement with Polymers
}

Nicolas François, David Lasne, Yacine Amarouchene, Brahim Lounis, Hamid Kellay

\section{To cite this version:}

Nicolas François, David Lasne, Yacine Amarouchene, Brahim Lounis, Hamid Kellay. Drag Enhancement with Polymers. Physical Review Letters, 2008, 100 (1), pp.018302. 10.1103/PhysRevLett.100.018302 . hal-00747122

\section{HAL Id: hal-00747122 \\ https://hal.science/hal-00747122}

Submitted on 20 Dec 2017

HAL is a multi-disciplinary open access archive for the deposit and dissemination of scientific research documents, whether they are published or not. The documents may come from teaching and research institutions in France or abroad, or from public or private research centers.
L'archive ouverte pluridisciplinaire HAL, est destinée au dépôt et à la diffusion de documents scientifiques de niveau recherche, publiés ou non, émanant des établissements d'enseignement et de recherche français ou étrangers, des laboratoires publics ou privés. 


\title{
Drag Enhancement with Polymers
}

\author{
N. François, D. Lasne, Y. Amarouchene, B. Lounis, and H. Kellay \\ Université Bordeaux 1, Centre de Physique Moléculaire Optique et Hertzienne (UMR 5798 CNRS), \\ 351 cours de la Libération, 33405 Talence, France
}

(Received 6 March 2007; revised manuscript received 27 June 2007; published 9 January 2008)

\begin{abstract}
Drag on a cylinder can be enhanced in the presence of polymers. This enhancement is directly related to the interaction of the polymers with the flow around the obstacle. The presence of the polymers modifies the flow in the vicinity of the cylinder giving rise to a band of higher shear, large molecular elongations and large velocity fluctuations. The measured drag on the cylinder is directly related to the modification of the flow field by the presence of the polymers.
\end{abstract}

DOI: 10.1103/PhysRevLett.100.018302

One of the most studied problems in hydrodynamics involving polymer solutions is drag reduction [1]. The addition of very small amounts of flexible polymers to a turbulent flow reduces the drag by over $50 \%$ opening a wide range of applications. While this effect has been known for decades, the exact mechanisms behind it remain ill understood. The key issue is not only how the polymer interacts with the flow but also what role the boundaries play. Recent theoretical progress points out that the role of the boundaries is crucial [2]. The influence of curved boundaries on the flow of polymer solutions is even more complex and has been shown to give rise to drag enhancement for spheres and cylinders [3-8]. The conformations of the polymers near the boundaries should play an essential role in determining the flow properties of polymer solutions. The conformation dynamics of single fluorescent polymer molecules have been studied in wellcontrolled flows $[9,10]$, in the flow around cylinders in electrophoretic mobility cells [11], and in some random flows through numerical simulations [12]. These studies point out that polymers may undergo strong elongation. The effect of the boundaries and the passage from single molecule dynamics to the behavior of solutions continues however to pose a certain number of problems such as the link between the polymer conformations near the walls and the macroscopic wall shear stress.

We here study the influence of polymers in the flow around a cylindrical obstacle in the Stokes regime (Reynolds number $\operatorname{Re} \ll 1$ ). The strength of the polymerflow interaction is quantified by the Weissenberg number $\mathrm{Wi}=\dot{\gamma} \tau$, where $\tau$ is the relaxation time of the polymer solution and $\dot{\gamma}$ is the deformation rate of the imposed flow. We have observed that the presence of polymers enhances the drag on a cylinder when Wi increases beyond a threshold value $\mathrm{Wi}_{\text {crit }}$. This enhancement is due to the existence of a strongly sheared region or shear band near the cylinder wall where the polymers show strong elongation and the velocity field exhibits large spatial fluctuations. The spatial extent of this shear band is less than $10 \mu \mathrm{m}$ showing that the flow modification occurring at the microscopic level gives rise to large modifications of the macroscopic drag. A
PACS numbers: 47.57.Ng, 47.57.Qk, 47.61.-k, 61.25.H-

consequence of these findings is that the presence of small cylinders on the walls of a pipe suppresses the ability of the polymers to reduce drag.

Two flow setups have been used. The first one is a square channel with a side $W=0.6 \mathrm{~cm}$ and a length of $20 \mathrm{~cm}$. The flow was produced by injecting fluid at constant flux. The cylinder used is an optical fiber of radius $R=30 \mu \mathrm{m}$ and length $l=0.3 \mathrm{~cm}$ which was fixed in the middle of one of the side walls in such a way that its free tip was placed at the center of the channel. The optical fiber was connected to a HeNe Laser so a light beam emanates from its free end. The optical fiber cantilever was developed to measure velocity in soap film experiments exhibiting quasi two dimensional flows [13] and we here extend its use to three dimensions to measure the drag force exerted by the flow. Most measurements of drag on cylinders and spheres in the presence of polymers had focused on measuring their terminal velocity as they fall through the solution. Here we measure the drag force precisely using the deflection of the fiber which is sensitive to forces as small as 100 nanoNewtons. Previous measurements by Verhelst and Nieuwstadt [5] had a precision of $0.1 \mathrm{~N}$ and used slightly shear thinning solutions. A CCD camera and a microscope objective were used to visualize the bright end of the fiber so as to measure its flow induced deflection. The polymer used was polyethylene oxide (PEO, $M_{w}=4 \times 10^{6} \mathrm{amu}$ and a contour length of roughly $38 \mu \mathrm{m}$ ), a highly flexible polymer. The polymer powder was dissolved, at different concentrations ranging from 50 to $3000 \mathrm{ppm}$ by weight ( $\mathrm{ppm})$, in deionized water. Sucrose was added to the solutions so as to be able to increase the viscosity of the solvent, making it shear independent, and increase the relaxation times of the polymer solutions. The test fluids were solutions of water and sucrose. The Newtonian test fluids and the PEO polymer solutions used have a constant viscosity $\eta$ versus shear rate. The viscosity measurements were carried out with a commercial rheometer in Couette geometry. The second setup is a microchannel of rectangular cross section. Soft lithography was used to construct $2 \mathrm{~cm}$ long, $0.1 \mathrm{~cm}$ wide and $160 \mu \mathrm{m}$ high PDMS (polydimethylsiloxane) microchannels with isolated $2 R=40 \mu \mathrm{m}$ diameter 
and $160 \mu \mathrm{m}$ high cylindrical obstacles embedded in the channel. Solutions of PEO seeded with a small amount of fluorescently labeled DNA molecules were studied in this part [9]. The solvent used is a buffer of $p \mathrm{H} 7$ with added sucrose. The DNA molecules were either $\lambda$-DNA or $T 4$ DNA with contour lengths of 20 and $68 \mu \mathrm{m}$, respectively. Since the conformation of the PEO molecules cannot be visualized directly, we use the DNA molecules as local probes of the polymer conformations and of the velocity field. The relaxation times of the DNA molecules are much larger than those of the PEO solutions in which they are embedded. We observed the DNA molecules using an inverted fluorescence microscope and a highly sensitive camera. Measurements were made at the center of depth of the channel $(h=80 \mu \mathrm{m})$, where the channel induced shear rate is negligible. The two parts of our experiment are complementary: the drag on the cylinder is measured using the fiber cantilever while the polymer conformations and the velocity field were obtained by visualizing the DNA molecules.

For the cylinder drag, the deflection of the fiber $d$ was measured for different flow rates. The drag force was then calculated as $F=\frac{3 \pi R^{4} E}{4 l^{3}} d$. The value of Young's modulus $E$ was taken as $72 \times 10^{9} \mathrm{~Pa}$. The shear stress $\sigma$ is obtained from the drag force as $\frac{F}{2 \pi R l}$. The average shear rate on the cylinder is given by $\dot{\gamma}=\frac{2 U}{R(\ln (W / 2 R)-1)}$ calculated by integrating the Poiseuille profile in the square duct over the length of the fiber using the expression for the drag force per unit length given in [14]. Here $U$ is the average velocity in the channel based on the imposed flux. In the test fluid, this shear stress is linear versus the shear rate with a slope given by the measured viscosity $\eta$. This shows that the fiber cantilever can be used as a viscometer. For the PEO polymer solutions, two regimes are observed. At low shear, $\sigma$ is linear versus $\dot{\gamma}$ with a slope given by the measured viscosity $\eta$ of the polymer solution. At shear rates higher than a threshold value $\dot{\gamma}_{c}$, the shear stress becomes nonlinear versus the shear rate and the measured drag is enhanced in comparison with an hypothetical linear behavior $\sigma=\eta \dot{\gamma}$. In Fig. 1, we plot $\frac{\sigma}{\eta \dot{\gamma}}$ versus $\frac{\dot{\gamma}}{\dot{\gamma}_{c}}$ where data from different concentrations of polymer are superimposed showing a flat region indicating linearity of the stress shear rate relation and a region which deviates from 1 signaling the onset of nonlinearity. The lower inset shows $\frac{\sigma}{\eta \dot{\gamma}}$ versus $\dot{\gamma}$ for the test solution: the result is flat for all examined shear rates as expected. The parameter $t_{c}=1 / \dot{\gamma}_{c}$ which describes the onset of nonlinearity can be compared to the relaxation time $\tau$ of the polymer solution [15]. For the dilute solutions below the entanglement concentration $c^{*}$, which is about $300 \mathrm{ppm}$ for the $4 \times 10^{6}$ molecular weight $\mathrm{PEO}, \tau$ is the Zimm relaxation time which is $5.5 \mathrm{~ms}$ [16]. For higher concentrations, $\tau$ increases with concentration. The measured $t_{c}$ follows the same trend as shown in the upper left inset of Fig. 1 where both $t_{c}$ and $\tau$ are plotted versus the PEO concentration. The ratio $\mathrm{Wi}_{\text {crit }}=\frac{\tau}{t_{c}}=\dot{\gamma}_{c} \tau$, corresponding to the transition from linear to nonlinear

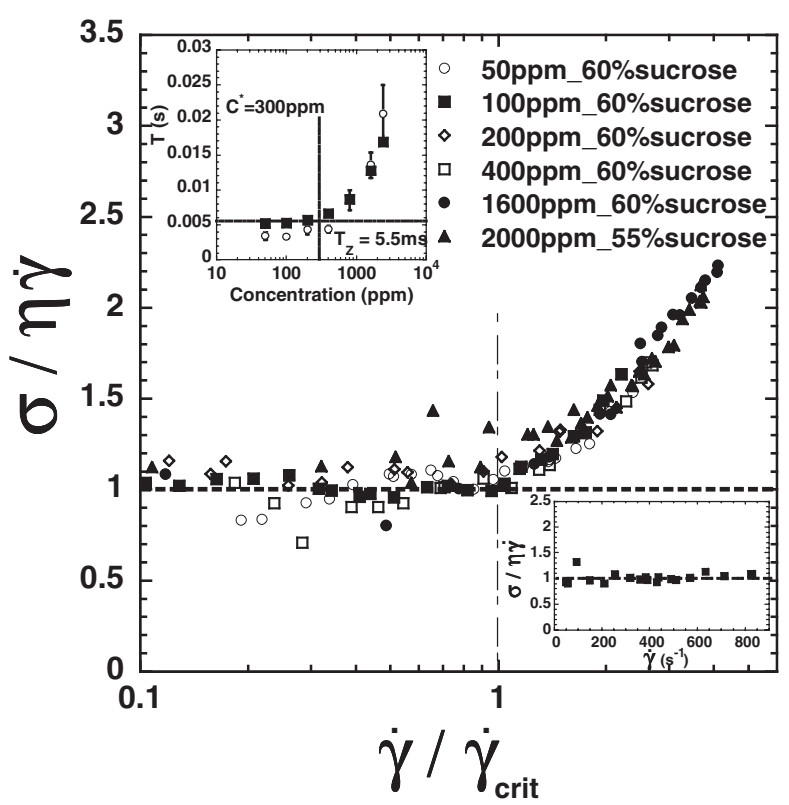

FIG. 1. Measurements of $\frac{\sigma}{\eta \dot{\gamma}}$ versus $\frac{\dot{\gamma}}{\dot{\gamma}_{c}}$ for polymer solutions at different concentrations obtained using the fiber cantilever; the lower inset shows the same quantity for the test solution. Upper inset: estimated relaxation times $\tau$ (filled squares) of the PEO solution and the critical time $t_{c}=1 / \dot{\gamma}_{c}$ (open circles) for the onset of nonlinearity plotted versus PEO concentration for $60 \%$ added sucrose.

drag, varies between 0.4 and 1.2. For values of Wi greater than 0.5 , one expects a coil-stretch transition to take place.

To examine the link between the drag enhancement and the coil-stretch transition, we used the second set up where we are able to visualize the conformations of the added fluorescently labeled DNA molecules. For values of $U<$ $U_{c}$, the DNA molecules appear as small bright dots and no stretching of the molecules is observed as seen in Fig. 2(a). However, for values of $U>U_{c}$, the DNA molecules stretch considerably but only near the cylinder wall as shown in Fig. 2(b). Far from the cylinder, no stretching is observed. A sequence of images showing the approach and the subsequent stretching of a single molecule around the cylinder for $U>U_{c}$ is seen in Fig. 2(c). When approaching the obstacle the molecule is only slightly extended until a minimal distance to the upstream stagnation point is reached where an abrupt extension occurs. As it travels along this region of maximum extension rate it gets stretched even more reaching maximal extension around $\theta=90^{\circ}$ [see Fig. 2(a) for the coordinate system], a region of simple shear flow, before starting to relax as it passes to the downstream side, a region of negative extension rate or contraction. The upstream stagnation point seems to play a key role in initializing the elongation of the molecules illustrating the role of curvature in the observed transition. A map of DNA mean lengths for $U>U_{c}$ is shown in Fig. 3(a). Large mean elongations are seen in a $10 \mu \mathrm{m}$ layer surrounding almost all the cylinder $\left(-150^{\circ} \leq \theta \leq 150^{\circ}\right)$. An asymmetry is also observed between the upstream and 


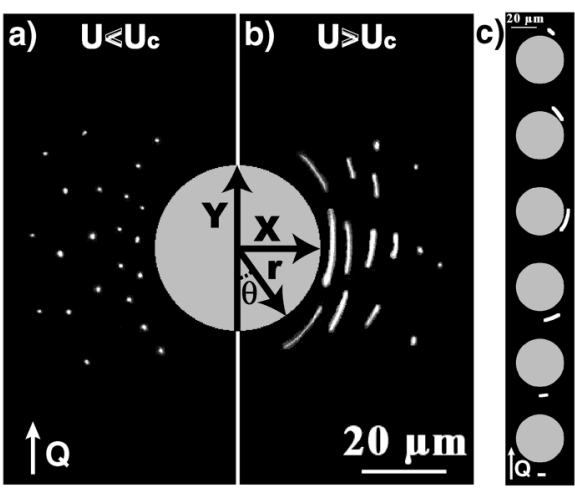

FIG. 2. Images of T4 DNA in the vicinity of the cylinder: (a) $U=0.3 U_{c}$ for which the DNA molecules remain as small bright dots of apparent radius $1.4 \mu \mathrm{m}$. (b) $U=6.2 U_{c}$ : Several DNA molecules at different locations from the $40 \mu \mathrm{m}$ cylinder showing highly elongated molecules. (c) A sequence of images showing the approach and elongation of a DNA molecule in the neighborhood of the cylinder.

downstream sides of the cylinder. While the elongation is large near the upstream wall, the downstream part exhibits low DNA elongation in the cylinder vicinity and a region of high elongation slightly downstream. The radial profile of the mean DNA length at $\theta=90^{\circ}$ is shown in Fig. 3(b). While the mean length is small and constant for $U=$ $0.3 U_{c}$, the mean polymer length for $U=6.2 U_{c}$ is large near the cylinder and decreases exponentially (indicated by the solid line) as the distance to the cylinder increases. The characteristic decay length is about $10 \mu \mathrm{m}$. Our results are in agreement with numerical simulations $[6,7]$ which show drag enhancement as well as strong elongation of the polymer molecules.

By tracking the DNA molecules we obtain their velocities and map out the velocity field around the cylinder. The velocity profile measured using latex beads for $U<U_{c}$ and $\theta=90^{\circ}$ is well represented by a Poiseuille flow between the cylinder wall and the lateral wall of the channel. At distances very close to the cylinder wall, the velocity profile extracted from tracking the DNA molecules is linear with the expected shear rate for our flow geometry as seen in Fig. 3(c). For $U>U_{c}$, the velocity profile near the wall splits into two linear regions as shown in Fig. 3(c) for $\theta=90^{\circ}$. Near the cylinder wall and within the characteristic decay length of the DNA elongation profile, a highly sheared layer appears, where the shear rate $\dot{\gamma}_{\text {wall }}$ is about twice the expected value. Away from this layer, the velocity varies roughly linearly with the distance $r$ with a shear rate comparable to the expected value. Hence the flow of the polymer around the cylinder seems to be composed of two bands or shear bands. It is interesting to note that the convective time at the border of the shear band (at $10 \mu \mathrm{m}$ from the cylinder wall) is roughly $1 \mathrm{~s}$ which is comparable to the relaxation time of the polymer molecules. The band delimits a region for which the convective time scale is larger than the characteristic time of the polymer. The inset of Fig. 3(c) shows that while far from the cylinder the histogram of DNA lengths shows a relatively sharp peak at a mean length of $3.3 \mu \mathrm{m}$, the histogram of DNA lengths near the cylinder is broader with a mean at $6.7 \mu \mathrm{m}$ and asymmetric in favor of large elongations. The DNA molecules are highly elongated in the high shear region near the cylinder wall. The velocity field also shows large fluctuations (up to 70\%) in this region as the map of $\frac{V-V_{\text {mean }}}{V_{\text {mean }}}$ depicts in the inset of Fig. 3(b) probably indicating the presence of elastic instabilities and the possibility of transition from a $2 \mathrm{D}$ creeping flow to a 3D flow [17]. Here, $\mathrm{V}$ is the velocity and $V_{\text {mean }}$ is the locally averaged velocity. Figure 4 shows that $\dot{\gamma}_{\text {wall }}$ takes values comparable to the expected shear for $U<U_{c}$ but rises faster than linear for $U>U_{c}$. This behavior is correlated with the drag enhancement found in the first experiment. Since the viscosity of the polymer solution in the high shear band is similar to that of the bulk solution because of the absence of shear thinning, these observations imply that the higher stress is related to the higher shear rate near the cylinder wall. A comparison between the increased drag as measured by the fiber and the in-
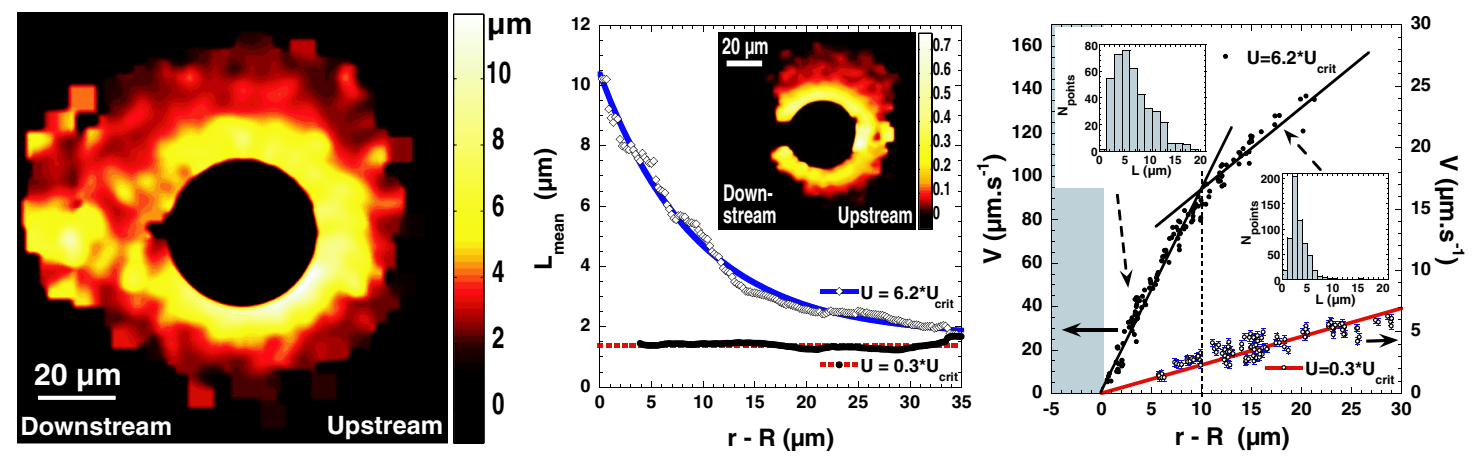

FIG. 3 (color online). (a) Map of DNA mean lengths (the levels are in $\mu \mathrm{m}$ ), obtained by averaging over $1 \mu \mathrm{m}^{2}$ bins, around the cylinder for $U=6.2 U_{c}$. (b) Plot of the mean elongation versus the distance from the cylinder for a wedge of $30^{\circ}$ centered around $\theta=90^{\circ}$ for $U=0.3 U_{c}$ and $U=6.2 U_{c}$. Inset: a map of $\frac{V-V_{\text {mean }}}{V_{\text {mean }}}$ around the cylinder for $U=3.1 U_{c}, V_{\text {mean }}$ is averaged over $1 \mu \mathrm{m}^{2}$ bins. (c) Velocity profiles versus distance $r$ from the cylinder for $U=0.3 U_{c}$ and $U=6.2 U_{c}$ for a wedge of $30^{\circ}$ centered around $\theta=90^{\circ}$. Upper and lower insets: histogram of DNA lengths in the high shear rate band and far away from the cylinder, respectively. 


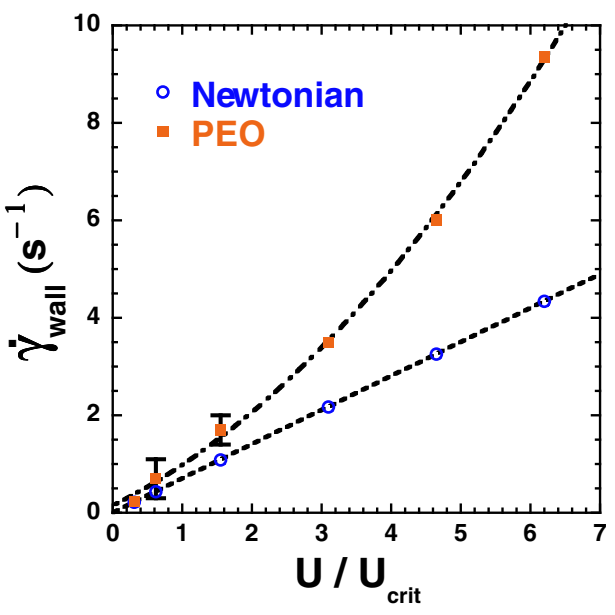

FIG. 4 (color online). Expected $\dot{\gamma}$ and measured $\dot{\gamma}_{\text {wall }}$ versus $U / U_{c}$ for $\theta=90^{\circ}$. Error bars indicate high fluctuations near the transition.

creased shear rate near the cylinder (Figs. 1 and 4) indicates that over $80 \%$ of the drag enhancement is given by the increased shear stress. The role of normal stresses is small mainly because of the circular geometry of the obstacle: their contribution comes from the slight asymmetry between the upstream and downstream parts.

The critical shear rate, at $\theta=90^{\circ}$, for the transition illustrated in Fig. 3 is $\dot{\gamma}_{c} \sim U_{c} / 2 R \sim 0.7 \pm 0.3 \mathrm{~s}^{-1}$ (the error is due to the difficulty of locating the transition as large fluctuations appear in its vicinity) which is comparable to the inverse relaxation time of the PEO solution $(\tau \sim 0.45 \mathrm{~s})$. The critical Weisenberg number turns out to be $\mathrm{Wi}_{\text {crit }} \sim 0.3 \pm 0.1$, which is roughly similar to the one determined for the concentrated PEO solutions in the drag measurements. Note that $\mathrm{Wi}_{\text {crit }}$ is the same for realizations with the $\lambda$ or T4 DNA molecules which have very different relaxation times, much greater than that of the PEO solution. These observations point out that the relevant time scale is that of the PEO solution and that the DNA molecules are acting as probes of the PEO conformations. The PEO molecules must be highly elongated in the vicinity of the wall just like their DNA counterparts which are behaving as passive probes of their conformations.

A direct consequence of the drag enhancement observed here is that in specially designed pipes drag reduction may be suppressed. To test this hypothesis we have designed a pipe on which several small cylinders were placed on the walls of the pipe. The diameter of the pipe was $2.2 \mathrm{~cm}$ while the diameter of the small cylinders was $0.4 \mathrm{~cm}$ with a height of $0.4 \mathrm{~cm}$. The small cylinders covered $5 \%$ of the pipe's surface. For a smooth pipe at a Reynolds number of 80000 , we measured a drag reduction of about $60 \%$ for a PEO concentration of $60 \mathrm{ppm}$. There was no drag reduction for the modified pipe under similar conditions for which we measured a slight drag enhancement of about $7 \%$. We checked that this was not due to polymer degradation since subsequent measurements using the smooth pipe and the same polymer solution indicated the presence of strong drag reduction immediately after the use of the solution in the modified pipe. This is a startling observation as it points out that the presence of curved boundaries (the small cylinders) affects drag reduction in an important way. Observations on the effects of wall roughness on drag reduction have already pointed out that wall roughness reduces drag reduction [1] but no full explanation has been given so far. Our results point out that the effect of wall roughness may be understood on the basis of increased drag due to the presence of curved boundaries.

In summary our study reveals that the coil-stretch transition occuring very near the cylinder walls is at the origin of the drag enhancement observed with flowing polymer solutions around cylindrical obstacles. The role of curved boundaries turns out to be crucial for this transition to take place as the upper stagnation point initiates the unwinding of the molecules. These findings have repercussions for the use of polymers in drag reduction since the presence of curved boundaries, as in porous media, for example, changes the structure of the flow near the walls and increases the apparent drag.

[1] B. A. Toms, in Proceedings of the International Congress on Rheology (North-Holland, Amsterdam, 1949); A. Gyr and H.W. Bewersdorff, Drag Reduction of Turbulent Flows by Additives (Kluwer, Dordrecht, 1995).

[2] V. Lvov et al., Phys. Rev. Lett. 92, 244503 (2004).

[3] R. P. Chhabra, K. Rami, and P. H. T. Uhlherr, Chem. Eng. Sci. 56, 2221 (2001).

[4] M. J. Solomon and S. J. Muller, J. Non-Newtonian Fluid Mech. 62, 81 (1996).

[5] J. M. Verhelst and F. T. M. Nieuwstadt, J. Non-Newtonian Fluid Mech. 116, 301 (2004).

[6] A. W. Liu et al., J. Non-Newtonian Fluid Mech. 77, 153 (1998).

[7] J.Sun et al., J. Non-Newtonian Fluid Mech. 86, 281 (1999).

[8] G. H. McKinley, in Transport Processes in Bubbles, Drops and Particles, edited by R. Chhabra and D. De Kee (Taylor \& Francis, London, 2001).

[9] T. T. Perkins, D. E. Smith, and S. Chu, Science 276, 2016 (1997).

[10] D. E. Smith, H. P. Babcock, and S. Chu, Science 283, 1724 (1999).

[11] G. C. Randall and P. S. Doyle, Phys. Rev. Lett. 93, 058102 (2004).

[12] A. Celani, A. Puliafito, and D. Vincenzi, Phys. Rev. Lett. 97, 118301 (2006).

[13] H. Kellay, X. L. Wu, and W. I. Goldburg, Phys. Rev. Lett. 74, 3975 (1995); M. Rivera et al., Rev. Sci. Instrum. 69, 3215 (1998).

[14] H. Faxèn, Proceedings of the Royal Swedish Academy of Engineering Sciences Vol. 187, p. 1, 1946.

[15] R. G. Larson, The Structure and Rheology of Complex Fluids (Oxford University, New York, 1999).

[16] P. G. de Gennes, Scaling Concepts in Polymer Physics (Cornell University, Ithaca, 1979).

[17] A. H. Shiang et al., Exp. Fluids 28, 128 (2000). 